\title{
A polynomial-time approximation algorithm for a geometric dispersion problem
}

\author{
Marc Benkert ${ }^{1 \star}$, Joachim Gudmundsson ${ }^{2}$, Christian Knauer ${ }^{3}$, Esther Moet $^{4}$, \\ René van Oostrum ${ }^{4}$, and Alexander Wolff ${ }^{1 \star}$ \\ 1 Faculty of Computer Science, Karlsruhe University, P.O. Box 6980, \\ D-76128 Karlsruhe, Germany. http://i11www.iti.uka.de/algo/group \\ 2 National ICT Australia Ltd $^{\ddagger}$, Sydney, Australia, \\ joachim.gudmundsson@nicta.com.au \\ 3 Institute of Computer Science, Freie Universität Berlin, Germany. \\ christian.knauer@inf.fu-berlin.de \\ 4 Department of Computing and Information Sciences, Universiteit Utrecht, \\ The Netherlands. \{esther, rene\}@cs.uu.nl
}

\begin{abstract}
We consider the problem of placing a maximal number of disks in a rectangular region containing obstacles such that no two disks intersect. Let $\alpha$ be a fixed real in $(0,1]$. We are given a bounding rectangle $P$ and a set $\mathcal{R}$ of possibly intersecting unit disks whose centers lie in $P$. The task is to pack a set $\mathcal{B}$ of $m$ disjoint disks of radius $\alpha$ into $P$ such that no disk in $\mathcal{B}$ intersects a disk in $\mathcal{R}$, where $m$ is the maximum number of unit disks that can be packed. Baur and Fekete showed that the problem cannot be solved in polynomial time for any $\alpha<1$, unless $\mathcal{P}=\mathcal{N} \mathcal{P}$. In this paper we present a polynomial time algorithm for $\alpha=2 / 3$.
\end{abstract}

\section{Introduction}

Obnoxious facility location problems consider the placement of facilities of which clients consider it undesirable to be in the proximity, for instance, nuclear power plants or garbage dumps. There are several models for and variations to the problem; see the survey by Cappanera [2]. Consider the following variant. Given a bounding rectangle $P$, a set $\mathcal{R}$ of $n$ points in $P$ (the red points), and an integer $k$, construct a set $\mathcal{B}$ of $k$ (blue) points such that the minimum distance from a blue point to another point (either red or blue) is maximized over all points in $\mathcal{B}$. If the optimal distance is denoted by $r_{\mathrm{opt}}$, then we can reformulate the problem as follows. Given a set of $n$ centers of possibly overlapping red disks with unknown radius $r_{\mathrm{opt}}$, determine $r_{\mathrm{opt}}$ and find a set of $k$ blue disks with radius $r_{\text {opt }}$ such that no blue disk overlaps any other disk, whether red or blue.

The problem of packing objects into a bounded region is one of the classic problems in mathematics and theoretical computer science, see for example the

\footnotetext{
* Supported by grant WO 758/4-1 of the German Science Foundation (DFG). Part of this work was done while M. Benkert visited NICTA on a DAAD grant.

$\ddagger$ Funded by the Australian Government's Backing Australia's Ability initiative, in part through the Australian Research Council.
} 
monographs $[10,12]$ which are solely devoted to this problem, and the survey by Tóth [11]. In this paper we consider problems related to packing disks into a polygonal region. As pointed out by Baur and Fekete in [1], even when the structure of the region and the objects are simple, only very little is known, see for example $[5,8]$.

Consider the following decision problem corresponding to our optimization problem. Given a set $\mathcal{R}$ of $n$ points in $P$, an integer $k$ and a radius $r$, decide whether $r>r_{\mathrm{opt}}$ or $r \leq r_{\mathrm{opt}}$. In the latter case, we must also give a set $\mathcal{B}$ of $k$ blue disk with radius $r$ such that no blue disk overlaps any other disk. If we had an algorithm at our disposal that solves the decision problem in polynomial time, then we could solve the original optimization problem in polynomial time by applying Megiddo's parametric search [9]. Unfortunately, the decision problem is known to be $\mathcal{N} \mathcal{P}$-complete [4]. Therefore we are looking for an algorithm that approximates the decision problem in the following sense. If $m$ disks of radius $r$ can be placed, then our algorithm places $m$ disks of radius $\alpha r$, for some fixed $\alpha \in(0,1]$. If $m<k$, then we know that $r>r_{\text {opt }}$, and if $m \geq k$, then either $r \leq r_{\mathrm{opt}}$, or $r>r_{\mathrm{opt}}$ and $\alpha r \leq r_{\mathrm{opt}}$. In other words, placement of at least $k$ disks of radius less than $\alpha r_{\text {opt }}$ is guaranteed.

Obviously, we would like to maximize $\alpha$ while keeping a polynomial running time. Given such an algorithm we can use it to compute an $\alpha$-approximation to the original optimization problem, again by using parametric search, albeit in a somewhat non-standard way.

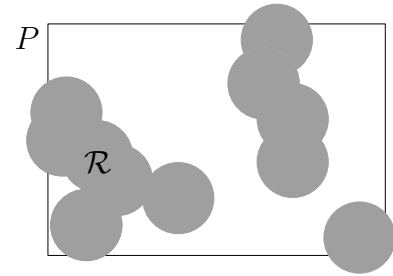

(a)

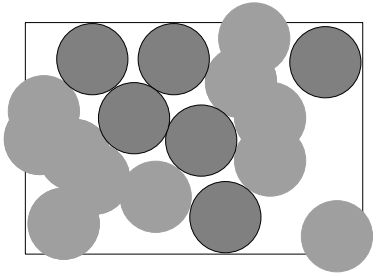

(b)

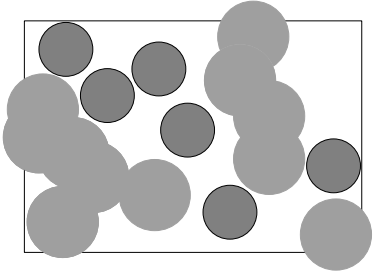

(c)

Fig. 1. (a) The input is a rectangle $P$ and a set of (possibly overlapping) unit disks $\mathcal{R}$. (b) An optimal solution where $m$ unit disks are packed. (c) Illustrating a 3/4approximation of $m$ disks (radius of the disks is $3 / 4$ ).

By rescaling $r$ to 1 , we can regard the decision problem as that of packing $m \geq k$ unit disks into a rectangle that is already partially covered by $n$ unit disks. In this paper, we consider the following problem (see Fig. 1):

Problem 1 (ApproxSize) Let $\alpha \in(0,1]$ be a fixed real. Given a bounding rectangle $P$ and a set $\mathcal{R}$ of possibly intersecting unit disks whose centers lie in $P$, pack at least $m$ non-intersecting disks of radius $\alpha$ into $P$, where $m$ is the maximal number of unit disks that can be packed in $P$. 
Note that we do not know the value of $m$ a priori. For $\alpha=1 / 2$, the problem can be solved by placing disks with radius $1 / 2$ greedily, i.e., as long as there is space to place a disk, we place one at an arbitrary feasible position. The following simple charging argument shows that we will place at least $m$ disks of radius $1 / 2$ in this way. Consider an arbitrary placement of $m$ unit disks, and charge a disk $C$ with radius $1 / 2$ to a unit disk $D$ if the center $c$ of $C$ lies inside $D$. After the greedy algorithm has finished, all of the $m$ unit disks have a charge of at least one. If not, we can place a disk with radius $1 / 2$ in an uncharged unit disk such that their centers coincide. This contradicts the termination condition of the greedy algorithm, thus the greedy algorithm is a $1 / 2$-approximation algorithm.

In their pioneering work [6] Hochbaum and Maas gave a polynomial-time approximation scheme (PTAS) for the problem of packing a maximal number of unit disks into a region. The problem is known to be $\mathcal{N} \mathcal{P}$-complete [4]. Even though the corresponding geometric dispersion problem looks very similar, inapproximability results have been shown. Baur and Fekete [1] proved hardness results for a variety of geometric dispersion problems, and their results can be modified to our setting with a bit of effort. For the case when the packed objects are unit squares, they show that APPRoxSize cannot be solved in polynomial time for any side length that exceeds $13 / 14$, unless $\mathcal{P}=\mathcal{N} \mathcal{P}$. By the same proof technique they also showed that APproxSize cannot be solved in polynomial time for any $\alpha<1$. Furthermore, for the case when the objects are squares, Baur and Fekete gave an $O\left(n^{38}\right)$-time 2/3-approximation algorithm. Our algorithm follows the same scheme of Baur and Fekete. First they compute a set $S$ of at least $2 / 3 \cdot m$ unit squares, using the algorithm by Hochbaum and Maas. Then they identify a set containing at least $m$ squares of side length $2 / 3$ with $S$.

However, because a square is a simpler shape and easier to place than a disk their arguments cannot be generalized to disks. The main contribution of this paper is a polynomial-time $2 / 3$-approximation algorithm.

ApproxSize has applications in non-photorealistic rendering system, see Fig. 2, where 3D models are to be rendered in an oil painting style, as well as in random examinations of, e.g., soil or water.

\section{Algorithm outline}

We now give a rough outline of our algorithm DiskPACKING, see Algorithm 1. We use the term $r$-disk as shorthand for a disk of radius $r$. For $r>0$ and a set $R \subseteq \mathbb{R}^{2}$ let the $r$-freespace of $R$, denoted $\mathcal{F}_{r}(R)$, be the set of the centers of all $r$-disks that are completely contained in $R$. By $\mathcal{F}_{r}^{\otimes}(R)$ we denote the Minkowski sum of $\mathcal{F}_{r}(R)$ and an $r$-disk.

We first compute the sets $\mathcal{F}_{1}=\mathcal{F}_{1}(P \backslash \bigcup \mathcal{R})$ and $\mathcal{F}_{1}^{\otimes}=\mathcal{F}_{1}^{\otimes}(P \backslash \bigcup \mathcal{R})$, see Fig. 3. Then, we apply the PTAS of Hochbaum and Maas [6] to $\mathcal{F}_{1}^{\otimes}$. For any positive integer $t$, the PTAS places at least $(1-1 / t)^{2} \cdot m$ unit disks into $\mathcal{F}_{1}^{\otimes}$ in $O\left(n^{t^{2}}\right)$ time, where $m$ is the maximum number of unit disks that can be packed into $\mathcal{F}_{1}^{\otimes}$ and $n$ is the minimum number of unit squares whose union covers $\mathcal{F}_{1}^{\otimes}$. Setting $t=26$ we obtain in $O\left(n^{676}\right)$ time a set $\mathcal{B}$ of $m^{\prime} \geq 12 / 13 \cdot m$ unit disks. 


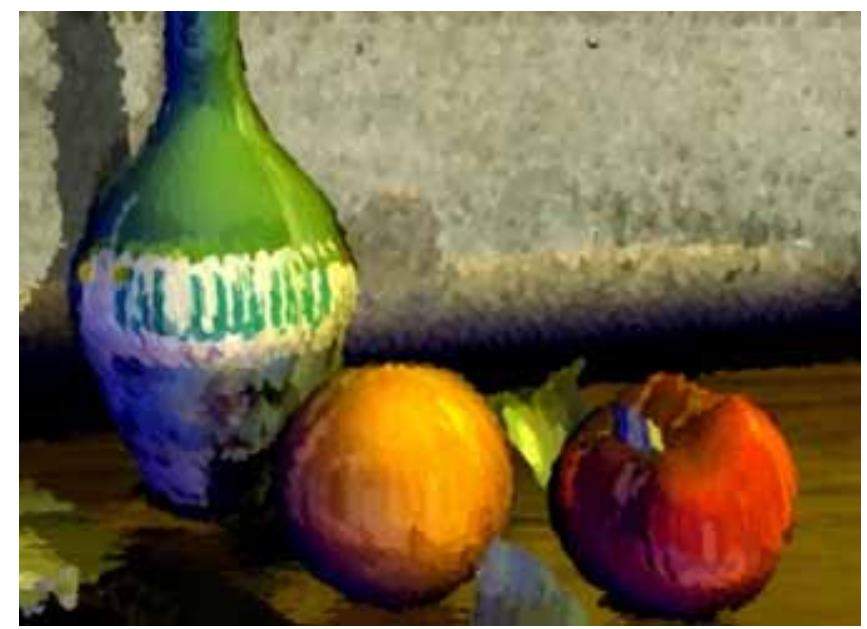

Fig. 2. The packing problem we consider in this paper occur in non-photorealistic rendering systems. For example, when a model should be rendered in an oil painting style.

Note that the approximation scheme by Hochbaum and Maas can be modified such that the algorithm is strongly polynomial with respect to the size of our input. If the number of disks that can be packed is not polynomial in the size of $P$ and $\mathcal{R}$ then there must exist a huge empty square region within $P$. This can be "cut out" and packed almost optimally by using a naïve approach. The added error obtained is bounded by $O\left(1 / \tilde{n}^{2}\right)$ where $\tilde{n}$ is the optimal number of disks that can be packed in the square. This step can be repeated until there are no more large empty squares. If the algorithm is required to output the placed disks then we have to add a term that is linear with respect to the number of placed disks.

Given $\mathcal{B}$ we compute a set $\mathcal{B}_{2 / 3}$ of disks of radius $2 / 3$ that has cardinality at least $13 / 12 \cdot m^{\prime} \geq m$ and is contained in $P \backslash \bigcup \mathcal{R}$. We obtain $\mathcal{B}_{2 / 3}$ in two steps. First, we compute a sufficiently large matching in the nearest-neighbor graph $G=(\mathcal{B}, E)$ of $\mathcal{B}$ with respect to a metric $\operatorname{dist}(\cdot, \cdot)$ that we will specify later. Second, we define a region for each pair of matching unit disks such that we can place three $2 / 3$-disks in each region (see Fig. 4) and all regions are pairwise disjoint. For each unmatched unit disk $D$ we place a $2 / 3$-disk $D^{\prime}$ such that the centers $d$ and $d^{\prime}$ of $D$ and $D^{\prime}$, respectively, coincide.

In the next sections we describe each step of Algorithm DiskPACKING in more detail.

\section{The freespace and a metric on unit disks}

We briefly recall the setting. We are given a set $\mathcal{R}$ of unit disks whose centers lie in a rectangle $P$, see Fig. 1(a). The disks in $\mathcal{R}$ are allowed to intersect. We 


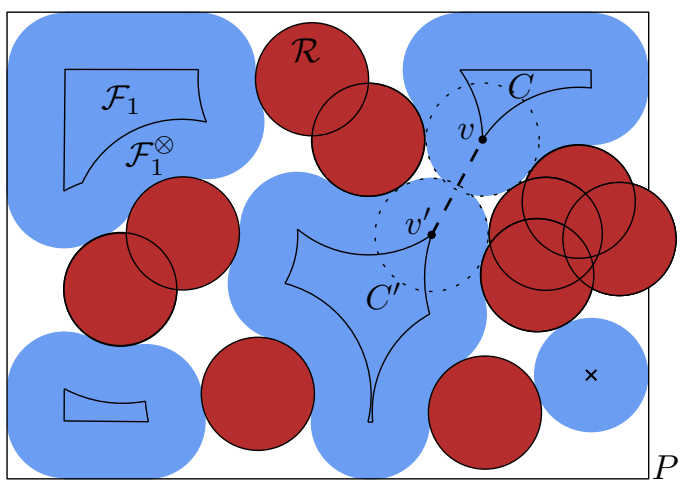

Fig. 3. The 1-freespace $\mathcal{F}_{1}^{\otimes}$ (light shaded) and a shortcut $v v^{\prime}$ (dashed) between the connected components $C$ and $C^{\prime}$ of $\mathcal{F}_{1}$.

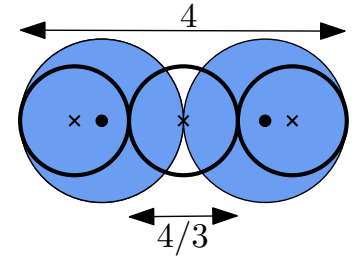

Fig. 4. Packing three 2/3-disks in the region spanned by a pair of unit disks.

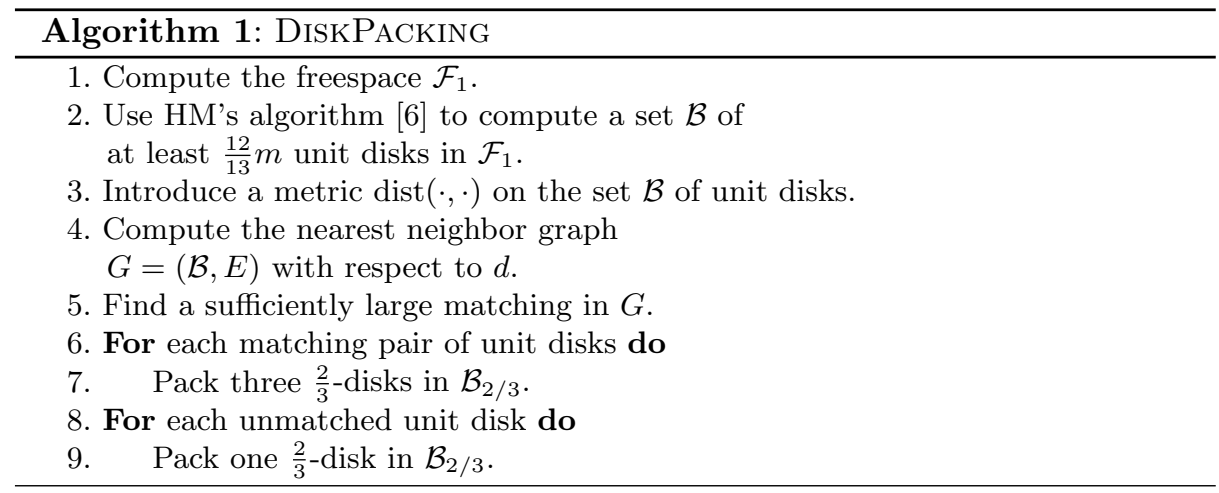

first compute the freespace $\mathcal{F}_{1}(P \backslash \bigcup \mathcal{R})$. According to Kedem et al. [7] the union of $s$ disks can be computed in $O\left(s \log ^{2} s\right)$ time and its complexity is linear in $s$. Applying their algorithm to the disks in $\mathcal{R}$ scaled by a factor of 2 and intersecting the resulting union with $P$ shrunk by 1 unit, we can compute $\mathcal{F}_{1}$ in $O\left(|\mathcal{R}| \log ^{2}|\mathcal{R}|\right)$ time, where $|\mathcal{R}|$ is the cardinality of $\mathcal{R}$.

Next, we want to introduce a metric $\operatorname{dist}(\cdot, \cdot)$ on unit disks in $\mathcal{F}_{1}^{\otimes}$. The idea of our algorithm is to use the connected components of $\mathcal{F}_{1}$ to identify all maximal regions where we can place $2 / 3$-disks. To guarantee that all such regions are discovered we need to join components of $\mathcal{F}_{1}$ that are not connected but still can hold 2/3-disks in the region between them. To make this possible we next define "shortcuts".

By $|p q|$ we denote the Euclidean distance between two points $p$ and $q$ in the plane.

Definition 1. Let $C$ and $C^{\prime}$ be two connected components of $\mathcal{F}_{1}$, and let $v$ and $v^{\prime}$ be vertices on the boundaries of $C$ and $C^{\prime}$, respectively. We say that the straight 
line segment $v v^{\prime}$ is a shortcut if $\left|v v^{\prime}\right| \leq \sqrt{11} \cdot 2 / 3 \approx 2.21$. Let $\mathcal{S}\left(C, C^{\prime}\right)$ be the set of all shortcuts induced by $C$ and $C^{\prime}$. We set

$$
\mathcal{F}_{1}^{+}=\mathcal{F}_{1} \cup \bigcup_{\substack{C, C^{\prime} \in \mathcal{F}_{1} \\ s \in \mathcal{S}\left(C, C^{\prime}\right)}} s .
$$

Figure 3 depicts $\mathcal{F}_{1}, \mathcal{F}_{1}^{\otimes}$, and a shortcut $v v^{\prime}$. Throughout the paper we will use upper-case letters to denote disks and the corresponding lower-case letters to denote their centers. Now, we are ready to define our metric for a connected component of $\mathcal{F}_{1}^{+}$, see Fig. 5(a)-(c).

Definition 2. Let $D$ and $D^{\prime}$ be unit disks in $\mathcal{F}_{1}^{\otimes}$, with centers $d$ and $d^{\prime}$, respectively. The distance $\operatorname{dist}\left(D, D^{\prime}\right)$ of $D$ and $D^{\prime}$ is the length of the geodesic $g\left(d, d^{\prime}\right)$ of $d$ and $d^{\prime}$ in $\mathcal{F}_{1}^{+}$. The tunnel $T\left(D, D^{\prime}\right)$ of $D$ and $D^{\prime}$ is the union of all points in $P \backslash \cup \mathcal{R}$ within distance 1 of a point on $g\left(d, d^{\prime}\right)$.

From the definition of $\mathcal{F}_{1}^{\otimes}$ it is easy to see that any $2 / 3$-disk $D_{2 / 3}$ centered at a point of $g\left(d, d^{\prime}\right)$ does not intersect any disk in $\mathcal{R}$. (This will also follow from Lemma 2.) Thus $D_{2 / 3}$ is contained in the tunnel $T\left(D, D^{\prime}\right)$. Since $\mathcal{R}$ is the union of a set of unit disks the geodesic between two points in $\mathcal{F}_{1}^{+}$can only consist of line segments and arcs of radius 2, see Fig. 5(b).

Recall that our algorithm will compute a matching in the nearest-neighbor graph $G=(\mathcal{B}, E)$ induced by the metric $\operatorname{dist}(\cdot, \cdot)$ on the set $\mathcal{B}$ of unit disks that we get from the PTAS by Hochbaum and Maas. For each pair $\left\{D, D^{\prime}\right\}$ in the matching we define a region $T_{2 / 3}\left(D, D^{\prime}\right)$ into which we will then place three $2 / 3$-disks as in Fig. 4 . An obvious way to define $T_{2 / 3}\left(D, D^{\prime}\right)$ would be to take the union of all 2/3-disks centered at points of the geodesic between $d$ and $d^{\prime}$ in $\mathcal{F}_{2 / 3}$. Our definition is unfortunately not that straight-forward, however our definition will simplify the proof of the main theorem in Section 4 . The theorem states that $T_{2 / 3}\left(D, D^{\prime}\right)$ and $T_{2 / 3}\left(F, F^{\prime}\right)$ are disjoint if $D, D^{\prime}, F$, and $F^{\prime}$ are pairwise disjoint. This is needed to ensure that the $2 / 3$-disks that we will place in the tunnels $T_{2 / 3}$ are disjoint.

Definition 3. Let $D$ and $D^{\prime}$ be unit disks in $\mathcal{F}_{1}^{\otimes}$. Let $g_{2 / 3}\left(d, d^{\prime}\right)$ be a geodesic from $d$ to $d^{\prime}$ in $\mathcal{F}_{2 / 3}\left(T\left(D, D^{\prime}\right)\right)$. The $2 / 3$-tunnel $T_{2 / 3}\left(D, D^{\prime}\right)$ of $D$ and $D^{\prime}$ is the union of all points in $P \backslash \cup \mathcal{R}$ within distance $2 / 3$ of a point on $g\left(d, d^{\prime}\right)$.

According to Chang et al. [3] the geodesics $g\left(d, d^{\prime}\right)$ and $g_{2 / 3}\left(d, d^{\prime}\right)$ from $d$ to $d^{\prime}$ can be computed in $O\left(|\mathcal{R}|^{2} \log |\mathcal{R}|\right)$ time.

\section{The nearest-neighbor graph}

Recall that $m$ is the maximum number of disjoint unit disks that fit in $\mathcal{F}_{1}^{\otimes}$. For $t=26$ the $(1-1 / t)^{2}$-approximation of Hochbaum and Maas [6] yields a set $\mathcal{B}$ of $m^{\prime} \geq 12 m / 13$ unit disks in $\mathcal{F}_{1}^{\otimes}$. Our idea is to compute the nearest-neighbor graph $G=(\mathcal{B}, E)$ induced by the metric $\operatorname{dist}(\cdot, \cdot)$, find a matching of sufficient 


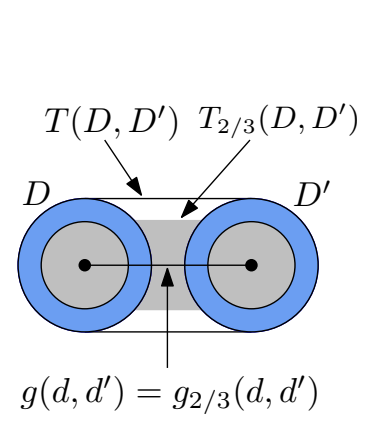

(a)

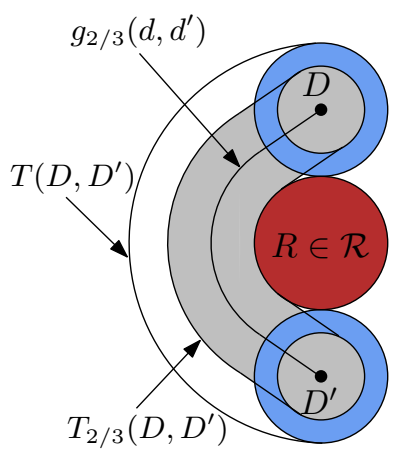

$g\left(d, d^{\prime}\right)=\widehat{g_{2 / 3}\left(d, d^{\prime}\right)}$

(b)

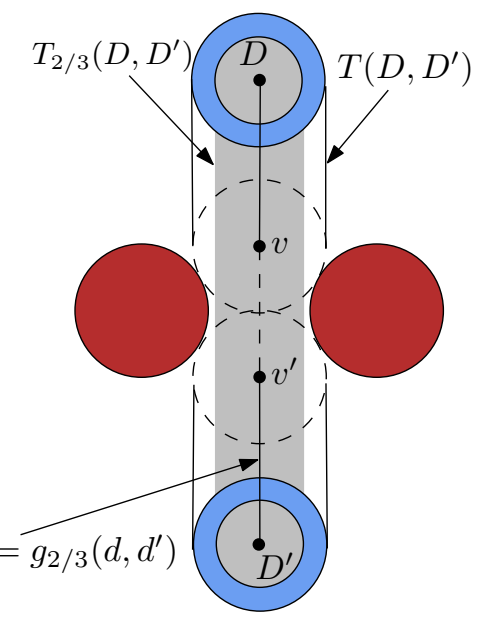

(c)

Fig. 5. Illustrating the geodesic $g\left(d, d^{\prime}\right)$ (a) in the unrestricted case, (b) in the case when obstacles are present and, (c) in the special case of a shortcut.

size in $G$, and finally place three $2 / 3$-disks in the $2 / 3$-tunnel $T_{2 / 3}(C, D)$ for each pair $\{C, D\}$ in the matching. We show that if we place another $2 / 3$-disk for each unmatched disk in $\mathcal{B}$, then we placed at least $13 m^{\prime} / 12 \geq m$ disks of radius $2 / 3$ in total.

By construction, two unit disks $D_{1}$ and $D_{2}$ whose centers lie in different components of $\mathcal{F}_{1}^{+}$have an empty intersection, so we can consider each connected component of $\mathcal{F}_{1}^{+}$separately.

Note that $G$ is a directed graph, where an edge $(C, D)$ is in $G$ if $D$ is the nearest neighbor of $C$, for $C, D \in \mathcal{B}$. In case of a tie, we pick any of the nearest neighbors of $C$, so every vertex in $G$ has only one outgoing edge.

After running the algorithm of Hochbaum and Maas we greedily add to $\mathcal{B}$ disjoint unit disks in $\mathcal{F}_{1}^{\otimes} \backslash \bigcup \mathcal{B}$ until no more disks can be added. This is needed to ensure the following lemma:

Lemma 1. The nearest-neighbor graph $G=(\mathcal{B}, E)$ (w.r.t. dist) is planar and has maximum degree 6 .

Proof. Let $C \in \mathcal{B}$ be an arbitrary unit disk, let $C^{\prime} \in \mathcal{B}$ be the nearest neighbor of $C$ in $\mathcal{B}$, and let $\mathcal{D}=\left\{D_{1}, \ldots, D_{k}\right\} \subseteq \mathcal{B}$ be the neighbors of $C$ in $\mathcal{B}$ for which $C$ is their nearest neighbor. If $k \leq 5$ then the degree bound obviously holds, thus we only have to consider the case when $k \geq 6$. For each disk $D_{i}, 1 \leq i \leq k$, place a unit disk $D_{i}^{\prime}$ with center on $g\left(c, d_{i}\right)$ such that $\left|c d_{i}^{\prime}\right|=2$, i.e., $D_{i}^{\prime}$ touches $C$, see Fig. 6. From the definition of the nearest-neighbor graph it follows that every point on $g\left(c, d_{i}\right)$ is closer to $C$ or $D_{i}$ than to any other unit disk in $\mathcal{B} \backslash\left\{C, D_{i}\right\}$. As a result the set $D_{1}^{\prime}, \ldots, D_{k}^{\prime}$ and $C^{\prime}$ has to be disjoint. Using a simple packing 
argument it follows that $k=6$ and that $C^{\prime} \in \mathcal{D}$, and thus the degree bound stated in the lemma holds.

Finally, $G$ is planar since no two edges in a nearest-neighbor graph can intersect.

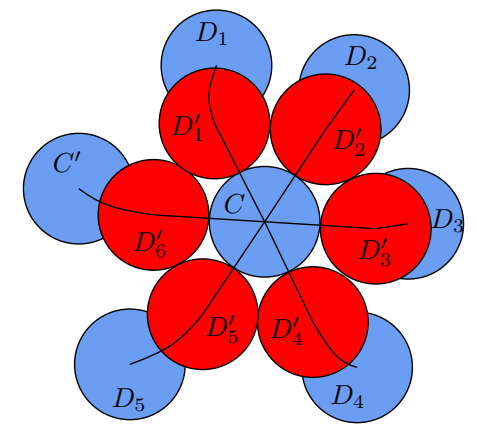

Fig. 6. Illustration for the proof of Lemma 1.

From now on we will call $\{C, D\} \subseteq \mathcal{B}$ a nearest pair if either $(C, D)$ or $(D, C)$ is an edge in $G$, i.e., either $D$ is the nearest disk in $\mathcal{B}$ to $C$ or $C$ is the nearest disk in $\mathcal{B}$ to $D$. For every nearest pair $\{C, D\}$ we define $\mathcal{A}(C, D)$ to be $C \cup D \cup T_{2 / 3}(C, D)$. As the nearest pair $\{C, D\}$ is a potential candidate to become a matching pair, we want to ensure that we can use $\mathcal{A}(C, D)$ to pack three $2 / 3$-disks in it such that all the packed $2 / 3$-disks are pairwise disjoint. Thus, we have to prove:

(i) three $2 / 3$-disks fit into $\mathcal{A}(C, D)$ and

(ii) for any nearest pair $\{E, F\}$ where $C, D, E$ and $F$ are pairwise disjoint $\mathcal{A}(C, D) \cap \mathcal{A}(E, F)=\emptyset$.

Note that we do not have to care whether $\mathcal{A}(C, D)$ intersects $\mathcal{A}(C, E)$ because the matching will choose at most one pair out of $\{C, D\}$ and $\{C, E\}$. Three $2 / 3$-disks obviously fit into $\mathcal{A}(C, D)$ since $C$ and $D$ do not intersect, thus, (i) is fulfilled. The remaining part of the paper will focus on proving (ii).

We split the proof into two parts. The first part (Lemma 4) shows that $T_{2 / 3}(C, D)$ does not intersect any disk other than $C$ and $D$. The second part (Theorem 1) shows that no two $2 / 3$-tunnels $T_{2 / 3}(C, D)$ and $T_{2 / 3}(E, F)$ intersect. We start with two technical lemmas that we need to prove the first part.

Lemma 2. Let $C$ and $D$ be two unit disks in $\mathcal{F}_{1}^{\otimes}$. If $|c d| \leq \frac{2}{3} \sqrt{11}$ then $g_{2 / 3}(c, d)$ is a straight line segment.

Proof. Let $T_{2 / 3}^{\prime}(C, D)$ be the Minkowski sum of a 2/3-disk and the line segment $c d$, see Fig. $7(\mathrm{a})$. If $g_{2 / 3}(c, d)$ is not a line segment, then a disk $E$ in $\mathcal{B} \cup \mathcal{R}$ 


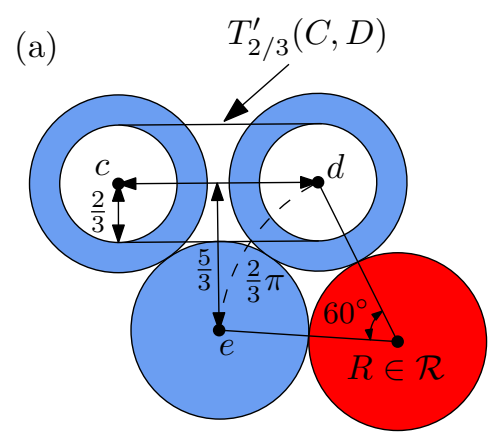

(b)

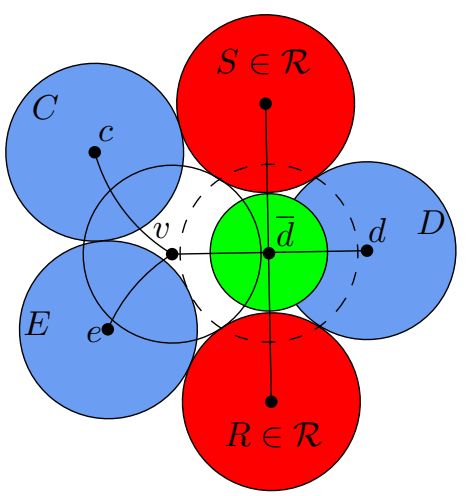

Fig. 7. Illustrations for (a) the proofs of Lemmas 2-3. (b) Illustrating case 2 in the proof of Lemma 4.

intersects $T_{2 / 3}^{\prime}(C, D)$. We establish a lower bound on $|c d|$ for this to happen. Note that $C, D$ and $E$ are pairwise disjoint as $C$ and $D$ are disks in $\mathcal{B}$.

Clearly, the minimum distance between $c$ and $d$ is attained if $E$ and $T_{2 / 3}^{\prime}(C, D)$ only intersect in a single point and furthermore, both $E$ and $C$ as well as $E$ and $D$ intersect in a single point. This means that $|c e|=|d e|=2$. Moreover, the Euclidean distance between $e$ and the straight-line segment $c d$ is $1+\frac{2}{3}=\frac{5}{3}$. By Pythagoras' theorem we calculate $|c d|$ to be at least $\frac{2}{3} \sqrt{11}$. This means that $T_{2 / 3}^{\prime}(C, D)$ is contained in $P \backslash \mathcal{R}$.

If $C$ and $D$ belong to different components of $\mathcal{F}_{1}$ then they must be connected via a shortcut according to Definition 1 . Thus, $g_{2 / 3}(c, d)$ is a line segment.

Lemma 3. Let $D$ and $E$ be two unit disks in $\mathcal{F}_{1}^{\otimes}$ that are infinitesimally close to each other. Then $\operatorname{dist}(D, E) \leq \frac{2}{3} \pi$.

Proof. For simplicity we assume that $D$ and $E$ touch, as illustrated in Fig. 7(a). The length of the curve $g(D, E)$ is maximized if there is an obstacle disk $R$ that touches $D$ and $E$ and no shortcut could be taken. In this case $g(D, E)$ describes a circular arc of radius 2 spanning $60^{\circ}$, thus its length is $\frac{1}{6} \cdot 2 \cdot 2 \pi=\frac{2}{3} \pi$.

Now, we are ready to prove the first part:

Lemma 4. Let $\{C, D\} \subseteq \mathcal{B}$ be a nearest pair. No disk of $\mathcal{B} \cup \mathcal{R} \backslash\{C, D\}$ intersects $T_{2 / 3}(C, D)$.

Proof. From the definition of freespace and Definitions 2 and 3 it immediately follows that neither $T(C, D)$ nor $T_{2 / 3}(C, D)$ are intersected by a disk in $\mathcal{R}$. Thus, it remains to prove that apart from $C$ and $D$ no disk in $\mathcal{B}$ intersects $T_{2 / 3}(C, D)$.

W.l.o.g. let $C$ be the disk in $\mathcal{R}$ closest to $D$. The proof is done by contradiction, i.e, we assume that there is a disk $E \in \mathcal{B}$ that intersects $T_{2 / 3}(C, D)$. 
First, we move a unit disk on $g(C, D)$ from the position of $D$ to the first position in which it hits $E$, denote the disk in this position by $\bar{D}$. We claim that the length of $g(\bar{d}, e)$, within $\mathcal{F}_{1}^{+}$, is shorter than $g(c, \bar{d})$. This obviously contradicts $C$ being the nearest neighbor of $D$, and would thereby complete the proof of the lemma.

We have to consider two cases for the upper bound on the length of $g(\bar{d}, e)$.

Case 1: If $\bar{d}$ is in $\mathcal{F}_{1}$ then the length of $g(\bar{d}, e)$ is maximized if there is an obstacle disk $R \in \mathcal{R}$ that touches $\bar{D}$ and $E$ and, $g(\bar{d}, e)$ is an arc, denoted $\mathcal{A}$, of radius 2 and spanning $60^{\circ}$. Lemma 3 yields $g(\bar{d}, e) \leq \frac{2}{3}$. It might be that $\mathcal{A}$ does not lie entirely within $\mathcal{F}_{1}$. However, as $\bar{D}, E \subseteq \mathcal{F}_{1}^{\otimes}$, there must then be a shortcut that would shorten the length of $g(\bar{d}, e)$ even further.

Next we give a lower bound on the length of $g(c, \bar{d})$. Since $E$ touches $T_{2 / 3}(C, D)$ and $\bar{D}$ it follows that $C$ and $\bar{D}$ are disjoint, otherwise $E$ could not intersect $T_{2 / 3}(C, D)$. Consequently $C, \bar{D}$ and $E$ are pairwise disjoint and, according to Lemma 2, the Euclidean distance between $c$ and $\bar{d}$ is greater than $\frac{2}{3} \sqrt{11}$. Putting the two bounds together we get:

$$
g(\bar{d}, e) \leq \frac{2}{3}<\frac{2}{3} \sqrt{11}<g(c, \bar{d}) .
$$

Case 2: If $\bar{d}$ is not in $\mathcal{F}_{1}$ then $\bar{d}$ must lie on a shortcut $v v^{\prime}$ and the unit disk $\bar{D}$ intersects at least one disk in $\mathcal{R}$. Let $v$ be the endpoint of the shortcut $\left(v, v^{\prime}\right)$ closest to $E$ and let $\bar{D}_{2 / 3}$ be the 2/3-disk centered at $\bar{d}$. Note that $v$ must lie in the same component as $e$, thus $g(\bar{d}, e)$ consists of a straight line segment from $\bar{d}$ to $v$ followed by the geodesic $g(v, e)$. The length of $g(\bar{d}, e)$ is maximized if the angle $\angle v \bar{d} e$ is maximized. This is the case if a unit disk $R \in \mathcal{R}$ touches $E$ and $\bar{D}_{2 / 3}$ and a unit disk $S \in \mathcal{R}$ touches $\bar{D}_{2 / 3}$ such that $\bar{d}, r$ and $s$ are collinear, as shown in Figure 7(b). By parametrization it follows that the length of $g(\bar{d}, e)$ is maximized if the length of $\bar{d} v$ is maximized, which is bounded by $1 / 3 \sqrt{11}$, according to Lemma 2 . By Pythagoras' theorem we can now compute the coordinates of $e$, they are $\approx(-1.8182,-0.8333)$, where the coordinate system is fixed by $\bar{d}=(0,0)$ and $r=(0,-5 / 3)$. The geodesic $g(v, e)$ consists of an arc of radius 2, applying the cosine theorem then yields that the length of $g(v, e)$ is approximately 1.1105 , which gives that $g(\bar{d}, e)<\frac{1}{3} \sqrt{11}+1.105$.

Next we need a lower bound on the length of $g(c, \bar{d})$. Using the same ideas as above, the position of $c$ can be computed by Pythagoras' theorem to be $\approx(-1.9356,1.1632)$ and the length of the geodesic $g(\bar{c}, v)$ is at least 1.4607 using the cosine theorem. Thus,

$$
g(\bar{d}, e) \leq \frac{1}{3} \sqrt{11}+1.105<\frac{1}{3} \sqrt{11}+1.4607<g(c, \bar{d}) .
$$

Since $g(\bar{d}, e)$ has been shown to be shorter than $g(c, \bar{d})$, in all cases, $\bar{d}$ must be closer to $c$ than to $e$, which is a contradiction to the initial assumption. This completes the proof of the lemma.

Note that the disks involved in this construction can be moved such that the lengths of $g(\bar{d}, e)$ and $g(c, \bar{d})$ changes. However, the above construction minimizes their difference. 
(a)

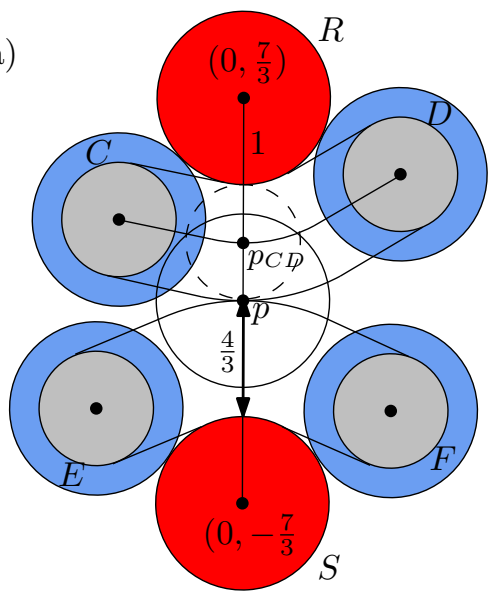

(b)

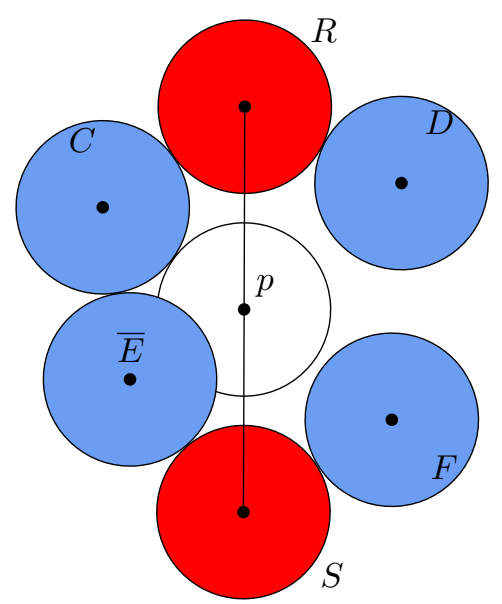

Fig. 8. Illustrating the proof of (a) Theorem 1 and (b) case (ii) in the proof of Theorem 1.

Lemma 4 proves that no other disks apart from $C$ and $D$ intersect $T_{2 / 3}(C, D)$. It remains to prove that no two $\frac{2}{3}$-tunnels $T_{2 / 3}(C, D)$ and $T_{2 / 3}(E, F)$ intersect.

Theorem 1. Let $\{C, D\},\{E, F\} \subseteq \mathcal{B}$ be two nearest pairs such that $C, D, E$ and $F$ are pairwise disjoint, it holds that $T_{2 / 3}(C, D) \cap T_{2 / 3}(E, F)=\emptyset$.

Proof. The proof is by contradiction again. Assume that $T_{2 / 3}(C, D)$ and $T_{2 / 3}(E, F)$ intersect.

Note that the geodesics $g_{2 / 3}(c, d)$ and $g_{2 / 3}(e, f)$ cannot intersect. If they did it would immediately follow that $g(c, d)$ and $g(e, f)$ also intersect. Let $i$ be one of the intersection points of $g(c, d)$ and $g(e, f)$. This implies that the lengths of $g(c, i), g(d, i), g(e, i)$ and $g(f, i)$ must all be equal. Then, however, either $g(c, d)$ or $g(e, f)$ could not be a geodesic-a contradiction. Thus, we an assume that $T_{2 / 3}(C, D)$ and $T_{2 / 3}(E, F)$ intersect but the do not cross. Note that this also includes the case when $g(c, d)$ and $g(e, f)$ follows the same shortcut. Thus, it is enough to prove the theorem for the case in which the tunnels intersect in a single point $p$, see Fig. 8(a).

Thus, $p$ lies in $\mathcal{F}_{1}$ and neither $g(c, d)$ nor $g(e, f)$ takes a shortcut containing $p$ and we can w.l.o.g. assume that no shortcut is taken at all. Again, we will show that $\{C, D\}$ and $\{E, F\}$ can not be nearest pairs at the same time. We observe that at least one of the disks $\{C, D, E, F\}$ intersects the unit disk $P$ with center $p$; otherwise there would be another disk in $\mathcal{B}$ located in the space between $C, D, E$ and $F$ which would immediately contradict $\{C, D\}$ as well as $\{E, F\}$ being nearest pairs. W.l.o.g. let $C$ be a disk that intersects $P$.

Let $p_{C D}$ be the point on $g_{2 / 3}(C, D)$ such that $\left|p p_{C D}\right|=2 / 3$, see Fig. 8(a). Define $p_{E F}$ correspondingly. We will assume that there is a vicinity of $p_{C D}$ and 
$p_{E F}$ in which $g_{2 / 3}(C, D)$ and $g_{2 / 3}(E, F)$ are arcs. The case when one vicinity of $p_{C D}$ and $p_{E F}$ is a straight-line is easier and can be handled using similar arguments.

The curvature of $g_{2 / 3}(C, D)$ and $g_{2 / 3}(E, F)$ in a vicinity of $p_{C D}$ and $p_{E F}$ induces the existence of two disks $R, S \in \mathcal{R}$ as illustrated in Fig. 8(a). Since $R$ and $S$ forces the curvature of $g_{2 / 3}(C, D)$ and $g_{2 / 3}(E, F)$ we may introduce the following coordinate system. The origin is $p$ and the coordinates of $r$ and $s$ are $\left(0, \frac{7}{3}\right)$ and $\left(0,-\frac{7}{3}\right)$, respectively.

As a consequence of Lemma 2 we get that $g_{2 / 3}(C, D)$ and $g_{2 / 3}(E, F)$ start with a straight-line segment of length at least $\frac{1}{3} \sqrt{11}$, again see Fig. 8(a). Thus, the curvature of $g_{2 / 3}(C, D)$ in $p_{C D}$ infers that $\left|c p_{C D}\right| \geq \frac{1}{3} \sqrt{11}$ holds, which means that $C$ either lies completely to the left of the $y$-axis or to the right. This holds analogously for the other disks. W.l.o.g. we assume that $C$ and $E$ lie to the left of the $y$-axis and $D$ and $F$ lie to the right, see Fig. 8(a).

Note that we have to take care which relationship inferred the pairs $\{C, D\}$ and $\{E, F\}$ are nearest pairs, e.g. $C$ could be the nearest neighbor of $D$ or $D$ could be the nearest neighbor of $C$. We will prove the following:

(i) $\operatorname{dist}(C, E)<\operatorname{dist}(E, F)$

(ii) $\operatorname{dist}(C, E)<\operatorname{dist}(C, D)$

(iii) $\operatorname{dist}(D, F)<\operatorname{dist}(C, D)$

Item (i) says that $C$ is closer to $E$ than $F$ is. Thus, in order for $\{E, F\}$ to be a nearest pair, $E$ must be the nearest neighbor of $F$. We use this fact to show that (ii) and (iii) hold. Together, (ii) and (iii) comprise the contradiction: (ii) says that $D$ is not the nearest neighbor of $C$, while (iii) says that $C$ is not the nearest neighbor of $D$. Hence, $\{C, D\}$ cannot be a nearest pair.

(i): To prove that $\operatorname{dist}(C, E)<\operatorname{dist}(E, F)$ we will argue that $T(E, F)$ intersects $C$, i.e. there is a unit disk $\bar{E}$ whose center lies on $g(E, F)$ that intersects $C$ and not $F$. Let $\bar{E}$ be defined by the left and bottommost point $\bar{e}$ on $g(E, F)$ such that $\bar{E}$ intersects $C$. This is illustrated in Fig. 8(b). The proof of (i) can then be completed by showing that $\operatorname{dist}(C, \bar{E})<\operatorname{dist}(\bar{E}, F)$.

First we prove that there exists a position of $\bar{e}$ such that $\bar{E}$ intersects $C$, i.e, a unit disk cannot pass between $C$ and $S$ without intersecting $C$. This could only be achieved by maximizing $|c s|$. Recall that $C$ intersects $P$, thus, $|c s|$ is maximized if $C$ touches $R$ and $P$, i.e. $C$ takes its left and topmost position, as shown in Fig. 8(b). Using Pythagoras' theorem we can compute the coordinates of $c$ for this setting to be $\approx(-1.62,1.17)$. From now on we will omit the sign $\approx$ when stating results of the calculations. Hence, it holds that $|c s| \leq 3.86$ which in turn yields that no unit disk can pass between $C$ and $S$ since this would require $|c s| \geq 4$.

Next, we try to minimize the distance $\operatorname{dist}(\bar{E}, F)$ in order to get $F$ to be closer to $\bar{E}$ than to $C$. For this $\bar{E}$ should take its rightmost position, which is attained if $C$ is as far as possible from $S$, i.e. takes position $(-1.62,1.17)$ again. Using Pythagoras' theorem, the coordinates of $\bar{e}$ is $(-1.29,-0.80)$. This means that $\left|\bar{e} p_{E F}\right| \geq 1.29$ and thus $\operatorname{dist}(\bar{E}, F) \geq 1.29+\frac{1}{3} \sqrt{11}$ as $\left|p_{E F} F\right| \geq \frac{1}{3} \sqrt{11}$ holds. 
According to Lemma $3 \operatorname{dist}(C, \bar{E}) \leq \frac{2}{3} \pi$, and we have:

$$
\operatorname{dist}(C, \bar{E}) \leq \frac{2}{3} \pi<1.29+\frac{1}{3} \sqrt{11} \leq \operatorname{dist}(\bar{E}, F),
$$

which concludes (i).

(ii): To prove that $\operatorname{dist}(C, E)<\operatorname{dist}(C, D)$ always holds we first establish a lower bound on $\operatorname{dist}(C, D)$ and then an upper bound on $\operatorname{dist}(C, E)$. For the lower bound on we try to push $C$ and $D$ as close as possible together under the restriction that $E$ can still be the nearest neighbor of $F$. To minimize $\operatorname{dist}(C, D)$, $C$ should take its right and bottommost position and $D$ should take its left and bottommost position.

For the bound on $D$ we only use that $D$ is not allowed to intersect the tunnel $T(E, F)$. If it did we would get $\operatorname{dist}(D, E)<\operatorname{dist}(E, F)$ by a similar argument as in (i). (Here, the corresponding point $\bar{f}$ can even lie further to the right than $(1.29,-0.80)$ as $D$ does not have to intersect $P$.) However, $\operatorname{dist}(D, E)<$ $\operatorname{dist}(E, F)$ together with (i) would immediately contradict $\{E, F\}$ to be a nearest pair. Disk $D$ takes its left and bottommost position without intersecting $T(E, F)$ if $D$ touches $R$ and is infinitesimal close to $T(E, F)$. For simplicity we assume that $D$ touches $T(E, F)$, see Fig. 9(a). Standard trigonometric calculations give the left and bottommost coordinates of $d$ to be $(1.70,1.29)$.

For the right and bottommost position of $C$ we use the following arguments. Let $\bar{f}$ be the rightmost point on $g(E, F)$ such that $\bar{F}$ touches either $C$ or $E$. We use that $E$ has to be touched by $C$ otherwise $C$ is closer to $F$ than $E$. We compute the right and bottommost position of $C$ if $\bar{F}$ touches $C$ and $E$ at the same time, see Fig. 9(b). Note, that this actually yields a position in which $C$ is closer to $F$ than $E$ (w.r.t. metric $d$ ). Again, standard trigonometry gives that the right and bottommost coordinates of $c$ is $(-1.35,0.86)$.

Now a lower bound on $|c d|$ is the Euclidean distance between $(1.70,1.29)$ and $(-1.35,0.86)$ which is 3.08 . We obtain the final lower bound on $\operatorname{dist}(C, D)$ by noting that both $C$ and $D$ touch $R$, if not the Euclidean distance between $c$ and $d$ could be shortened, hence the geodesic has to follow the circular arc around $R$, thus we get $\operatorname{dist}(C, D)>3.49$.

By Pythagoras' theorem we can also compute the coordinates of $\bar{f}$ to be $(0.25,-0.35)$ - we will need them in the proof of (iii).

To prove (ii) it remains to show an upper bound on $\operatorname{dist}(C, E)$ which is less than 3.49. We try to push $C$ and $E$ as far away from each other as possible, under the restriction that $E$ is still the nearest neighbor of $F$. It is clear that $C$ has to take its left and topmost position which we already know is $(-1.62,1.17)$, from (i), while $E$ should take its left and bottommost position. Again, we only use that on the rightmost point $\bar{f}$ on $g(E, F)$ such that either $C$ or $E$ is touched by $\bar{F}$, it must be $E$ that is touched. First, we compute the rightmost point $\bar{f}$ on $g(E, F)$ where $\bar{F}$ touches $C$ taking position $(-1.62,1.17)$ and touches $S$. As we know the coordinates of $c$ and $s$, we can compute the coordinates of $\bar{f}$ by Pythagoras' theorem. It holds that $\bar{f}$ is $(-0.33,-0.36)$, see Figure 10(a). Now, $E$ takes its left and bottommost position if it touches $S$ and $\bar{F}$. Using Pythagoras' again, we get that $e=(-1.87,-1.64)$. This yields the upper bound on $|c e|$ of 
(a)

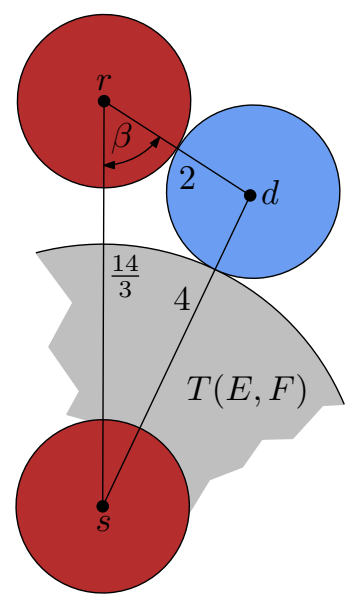

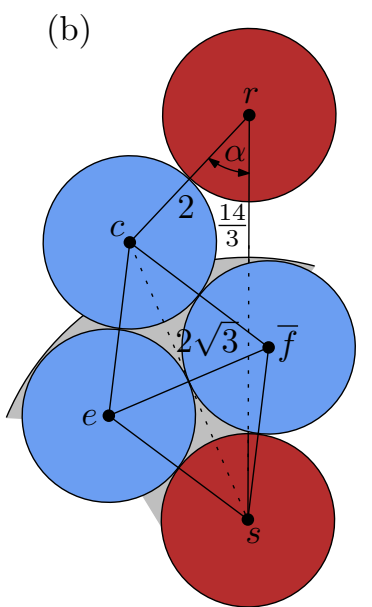

Fig. 9. Illustration of the proof of the lower bound on $|c d|$ in case (ii).

2.82. However, $g(C, E)$ may have to curve around $S$ so we get $\operatorname{dist}(C, E)<\pi$. Putting it together we get:

$$
\operatorname{dist}(C, E)<\pi<3.49<\operatorname{dist}(C, D),
$$

and we are done with (ii).

(iii): We use the lower bound on $\operatorname{dist}(C, D)$ that was derived in (ii). Thus, we only have to show an upper bound on $\operatorname{dist}(D, F)$ which is less than 3.49. For the upper bound we try to push $D$ and $F$ as far away from each other as possible, under the restriction that $E$ can still be the nearest neighbor of $F$. For this, $D$ has to take its right and topmost position while $F$ has to take its right and bottommost position. We can assume that $D$ takes position $(1.70,1.29)$, the position of $D$ which was responsible for the lower bound on $\operatorname{dist}(C, D)$. This assumption is justified since, if $D$ does not take position $(1.70,1.29)$, we move $D$ on $g(C, D)$ to this position, say $\bar{D}$, and show that $\operatorname{dist}(\bar{D}, F)<3.49$ Then, $\operatorname{dist}(D, F)<\operatorname{dist}(C, D)$ also holds since $\operatorname{dist}(C, \bar{D})>3.49$.

To maximize $\operatorname{dist}(D, F)$ we need $F$ to take its bottommost position. Consider the disk $P^{\prime}$ that touches $C$ and $S$ and lies to the right of $c s$, see Figure 10(b). For the right and bottommost position of $C$, which is $(-1.35,0.86)$, we already computed the position of $P^{\prime}$ to be $(0.25,-0.35)$. This implies that $D$ does not intersect $P^{\prime}$ as $\left|d p^{\prime}\right|>2$ (recall that the position of $D$ was decided in the previous section). We have shown that $C, D$ and $E$ do not intersect $P^{\prime}$, however, then $F$ has to intersect $P^{\prime}$ otherwise there would be another disk in $\mathcal{B}$ located in the space between $C, D, E$ and $F$ which would immediately contradict $\{C, D\}$ as well as $\{E, F\}$ being nearest pairs. The disk $F$ now takes its right and bottommost position if it touches $P^{\prime}$ and $S$. Using Pythagoras' theorem the position of $F$ is shown to be $(1.84,-1.55)$. 
As a result we get $|d f|<2.84$ but since $g(D, F)$ may have to curve around some other disk we get $\operatorname{dist}(D, F)<3.18$. Putting it together we get:

$$
\operatorname{dist}(D, F)<3.18<3.49<\operatorname{dist}(C, \bar{D}) \leq \operatorname{dist}(C, D),
$$

and we are done with (iii) and, hence also the theorem.
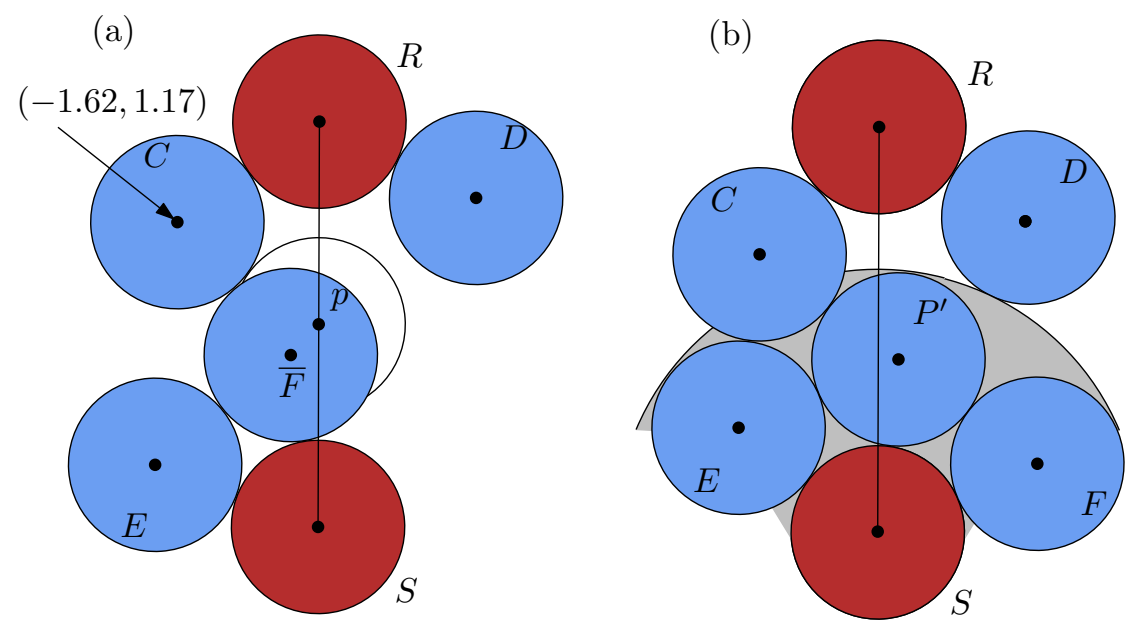

Fig. 10. (a) The upper bound on $|c e|$ in case (ii). (b) Illustrating the proof of case (iii).

\section{The set $\mathcal{B}_{2 / 3}$}

After computing $\mathcal{B}$ and the nearest neighbor graph $G=(\mathcal{B}, E)$, we compute a matching in $G$. Let $m^{\prime}=|\mathcal{B}|$ be the number of unit disks in $\mathcal{B}$. We show that we can find a matching in $G$ where the number of matched disks is at least $\frac{1}{6} \cdot m^{\prime}$. Observe that $G$ can consist of more than one connected component. We look at each connected component, denoted $C$, separately. From Lemma 1 it follows that $C$ contains a spanning tree of degree at most 6 . It is easy to see that there is a matching in $C$ that matches at least $\frac{1}{6} \cdot c$ disks, where $c$ is the number of disks in $C$. Doing this for each connected component yields a matching in $G$ that contains at least $\frac{1}{6} \cdot m^{\prime}$ matched disks.

According to Theorem 1 and Lemma 4 we can pack three 2/3-disks in $\mathcal{A}(C, D)$ for every matched pair $\{C, D\}$ such that these $2 / 3$-disks are pairwise disjoint. For each of the remaining unmatched disks we pack one $2 / 3$-disk in each disk. The set of all the placed disks is denoted $\mathcal{B}_{2 / 3}$. By construction, there are no interferences between these sets belonging to different connected components of $\mathcal{F}_{1}^{+}$. Thus, the cardinality of $\mathcal{B}_{2 / 3}$ is at least $\frac{1}{6} \cdot \frac{3}{2} \cdot m^{\prime}+\frac{5}{6} \cdot m^{\prime}=\frac{13}{12} \cdot m^{\prime}$. Since 
the cardinality of $\mathcal{B}$ is at least $\frac{12}{13} \cdot m$, the set $\mathcal{B}_{2 / 3}$ contains at least $m 2 / 3$-disks and we can conclude with the following theorem:

Theorem 2. Algorithm DiskPACKING is a polynomial-time 2/3-approximation for the problem APPROXSize.

\section{Conclusion}

Naturally our result is purely of theoretic interest. The bottleneck for the running time is the application of Hochbaum and Maas' PTAS with approximation factor $12 / 13$. To obtain an algorithm with better running time, it seems unavoidable to use a completely different approach. For future work it would also be desirable to narrow the gap between the approximation factor of $2 / 3$ of our algorithm and the inapproximability result of Baur and Fekete [1]. We conjecture that, unless $\mathcal{P}=\mathcal{N} \mathcal{P}$, the lower bound of $2 / 3$ is indeed tight. However, by Baur and Fekete's approach one gets only an inapproximability result which is slightly smaller than 1.

\section{References}

1. C. Baur and S. P. Fekete. Approximation of Geometric Dispersion Problems. Algorithmica, 30:450-470, 2001.

2. P. Cappanera. A survey on obnoxious facility location problems. Technical Report TR-99-11, University of Pisa, 1999.

3. E.-C. Chang, S. W. Choi, D. Kwon, H. Park and C.-K. Yap. Shortest path amidst disc obstacles is computable. In proceedings of the 21st ACM Symposium on Computational Geometry, 2005.

4. R. J. Fowler, M. S. Paterson and S. L. TAnimoto. Optimal packing and covering in the plane are NP-compete. Information Processing Letters, 12:133$137,1981$.

5. Z. FüREDI. The densest packing of equal circles into a parallel strip. Discrete $\&$ Computational Geometry, 6:95-106, 1991.

6. D. Hochbaum And W. MaAs. Approximation Schemes for Covering and Packing Problems in Image Processing and VLSI. Journal of the ACM, 32:130-136, 1985.

7. K. Kedem, R. Livne, J. Pach And M. Sharir. On the union of Jordan regions and collision-free translational motion admidst polygonal obstacles. Discrete $\mathcal{G}$ Computational Geometry, 1:59-71, 1986.

8. C. Maranas, C. Floudas and P. Pardalos. New results in the packing of equal circles in a square. Discrete Mathematics, 128:187-293, 1995.

9. N. Megiddo. Applying parallel computation algorithms in the design of serial algorithms. Journal of the ACM, 30(4):852-865, 1983.

10. C. A. Rogers. Packing and Covering. Cambridge University Press, 1964.

11. G. F. То́тн. Packing and Covering. In Handbook of Discrete and Computational Geometry, 2nd Edition J. E. Goodman and J. O'Rourke, editors CRC Press LLC, 2004.

12. C. Zong And J. Talbot. Sphere Packings. Springer-Verlag, 1999. 